\title{
Acid-Responsive Polymeric Doxorubicin Prodrug Nanoparticles \\ Encapsulating a Near-Infrared Dye for Combined \\ Photothermal-Chemotherapy
}

Yuanyuan Zhang, ${ }^{\dagger}, \Delta$ Cathleen Teh, ${ }^{\S, \Delta}$ Menghuan Li, ${ }^{\|}$Chung Yen Ang, ${ }^{\dagger}$ Si Yu Tan, ${ }^{\dagger}$ Qiuyu Qu, ${ }^{\dagger}$ Vladimir Korzh, ${ }^{*}, \S$ and Yanli Zhao ${ }^{*}, \dagger, \|$

${ }^{\dagger}$ Division of Chemistry and Biological Chemistry, School of Physical and Mathematical Sciences, Nanyang Technological University, 21 Nanyang Link, 637371, Singapore

${ }^{\$}$ School of Chemical Engineering, Huaihai Institute of Technology, Lianyungang 222005, P. R. China

${ }^{\S}$ Zebrafish Translational Unit, Institute of Molecular and Cell Biology, 61 Biopolis Drive, 138673,

Singapore

"School of Materials Science and Engineering, Nanyang Technological University, 639798, Singapore

${ }^{\Delta}$ These authors (Y.Z. and C.T.) contributed equally to this work.

*Corresponding author: zhaoyanli@ntu.edu.sg; vlad@imcb.a-star.edu.sg 

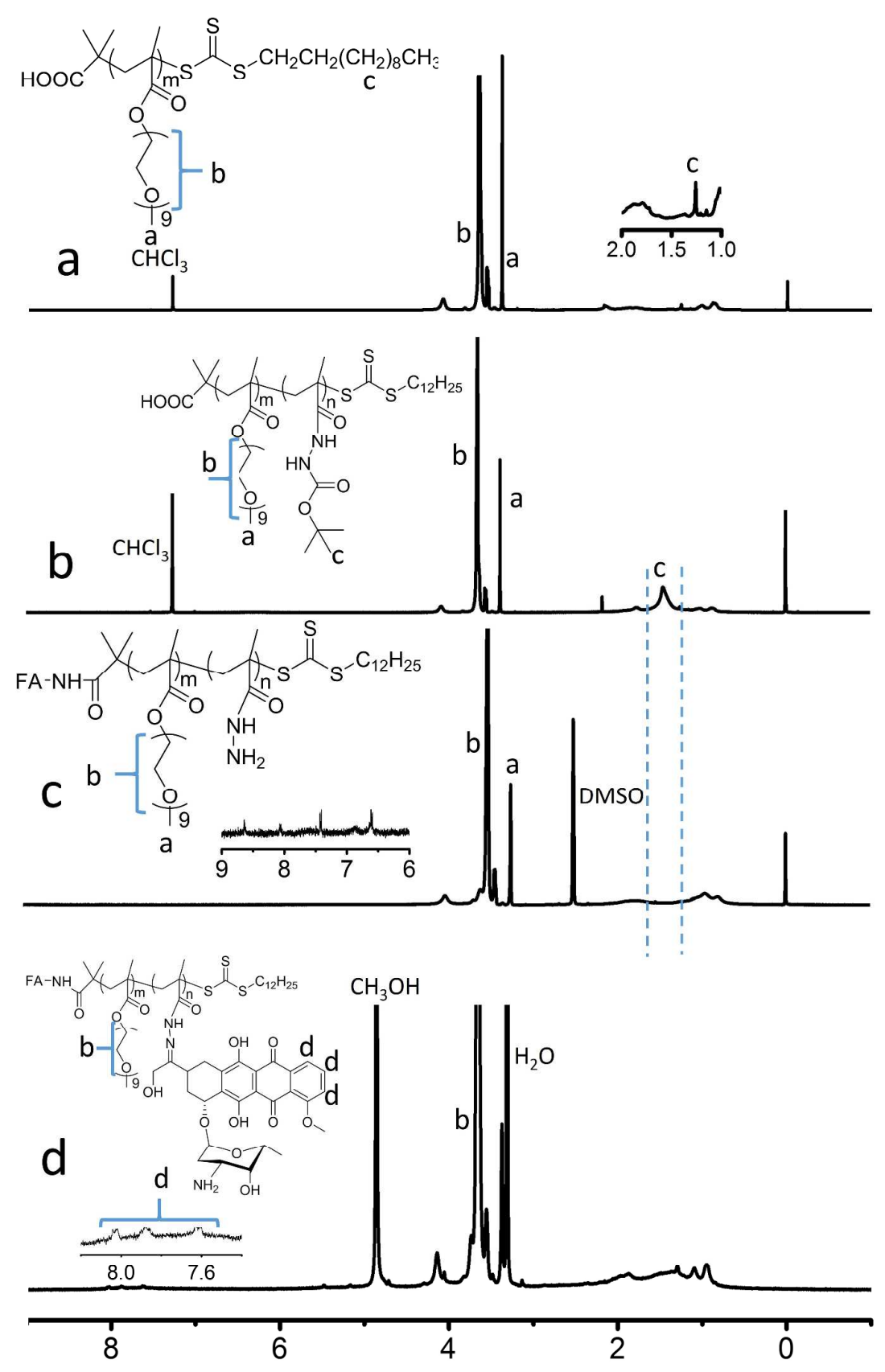

Figure S1. ${ }^{1} \mathrm{H}$ NMR spectra of (a) POEGMA in $\mathrm{CDCl}_{3}$, (b) PMABH- $b$-POEGMA in $\mathrm{CDCl}_{3}$, (c) $\mathrm{PNHNH}_{2}-b$-POEGMA-FA in DMSO- $d_{6}$, and (d) PDOX in MeOD. 
Table S1. Molecular weights and molecular weight distributions of POEGMA macro-RAFT agent and PMABH- $b$-POEGMA diblock copolymer.

\begin{tabular}{ccccc}
\hline Polymer & $\begin{array}{c}M_{\mathrm{n}(\mathrm{th})}{ }^{a} \\
\mathrm{~g} \mathrm{~mol}^{-1}\end{array}$ & $\begin{array}{c}M_{\mathrm{n}(\mathrm{NMR})}{ }^{b} \\
\mathrm{~g} \mathrm{~mol}^{-1}\end{array}$ & $\begin{array}{c}M_{n(G P C)}{ }^{c} \\
\mathrm{~g} \mathrm{~mol}^{-1}\end{array}$ & $M_{\mathrm{w}} / M_{\mathrm{n}}{ }^{c}$ \\
\hline $\begin{array}{c}\text { POEGMA } \\
\text { PMABH- } b \text {-POEGMA }\end{array}$ & 9700 & 9900 & 9100 & 1.16 \\
\hline
\end{tabular}

${ }^{a}$ Theoretical molecular weight. ${ }^{b}$ Calculated from ${ }^{1} \mathrm{H}$ NMR spectra. ${ }^{c}$ Determined by GPC.

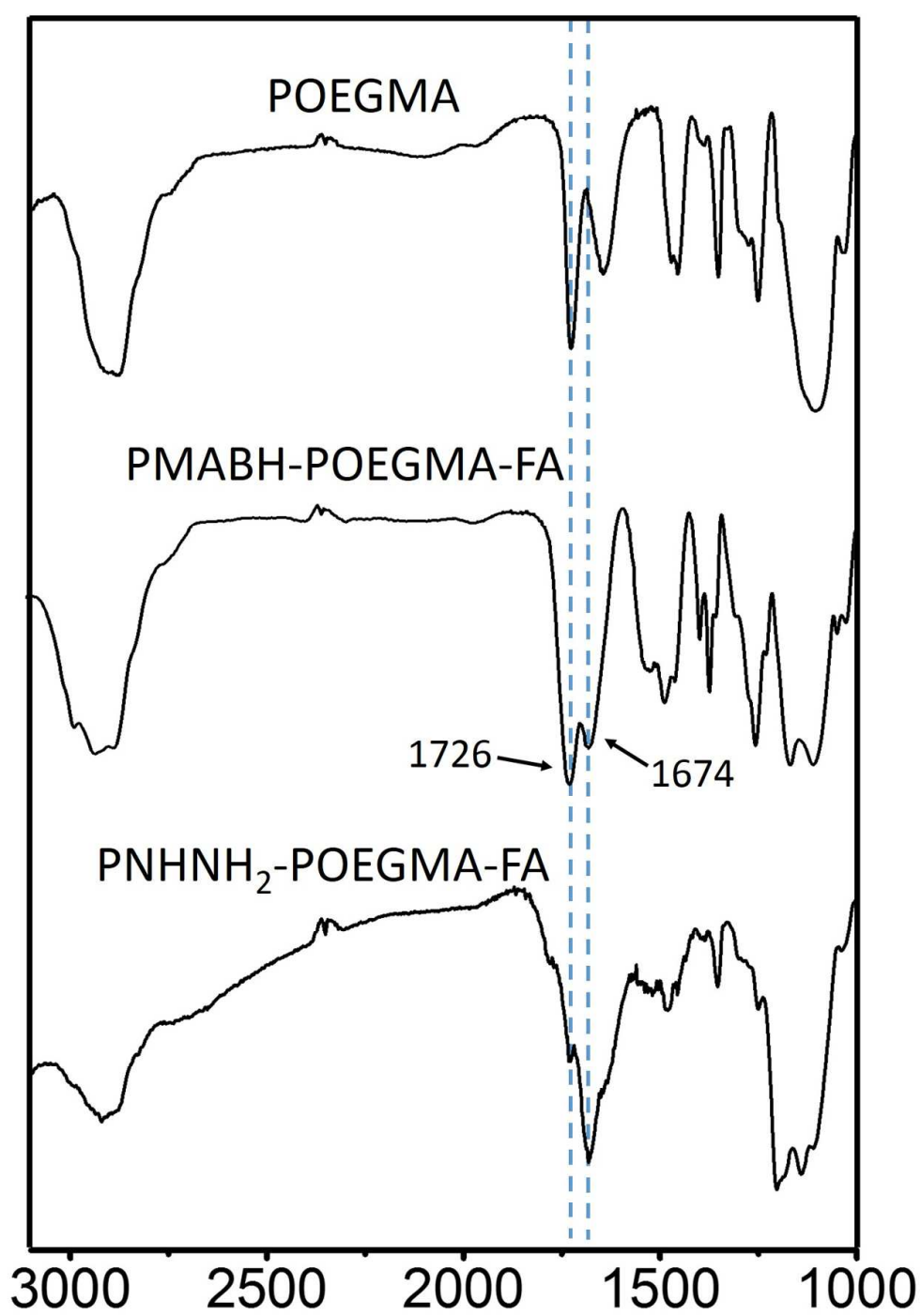

Figure S2. FT-IR spectra of POEGMA, PMABH-POEGMA-FA, and PNHNH 2 -POEGMA-FA. 


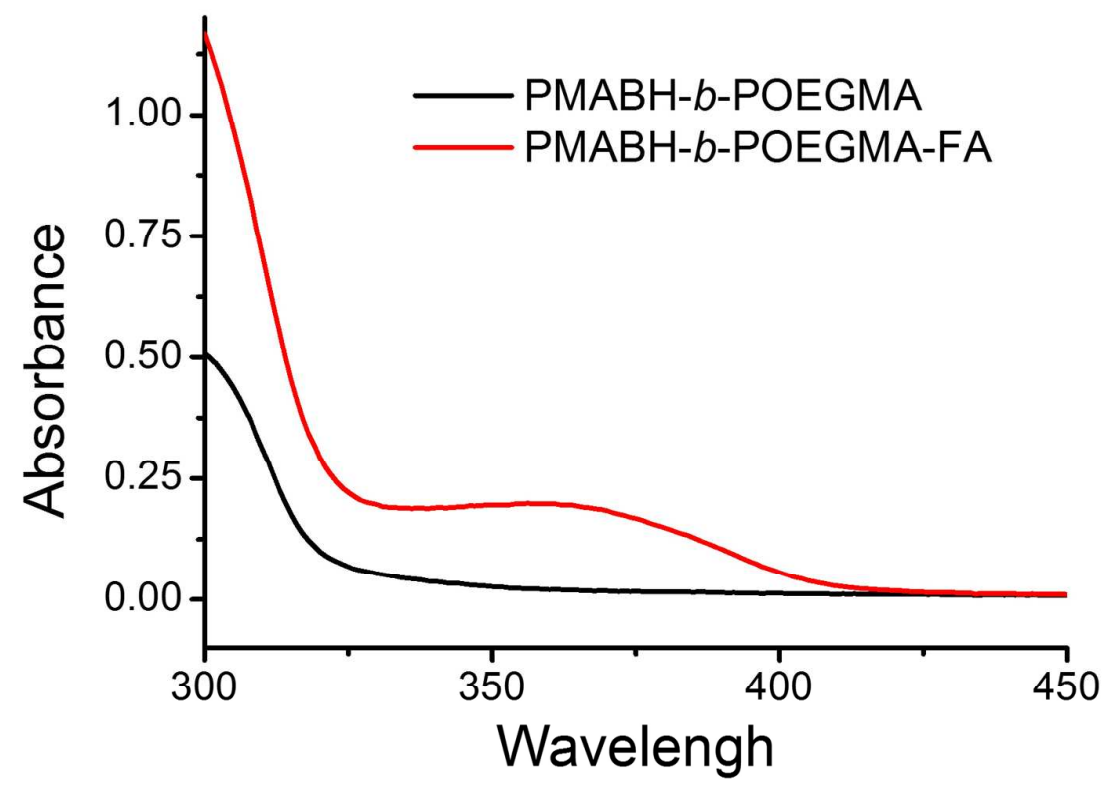

Figure S3. UV-Vis absorption spectra of PMABH- $b$-POEGMA and PMABH- $b$-POEGMA-FA in DMSO.

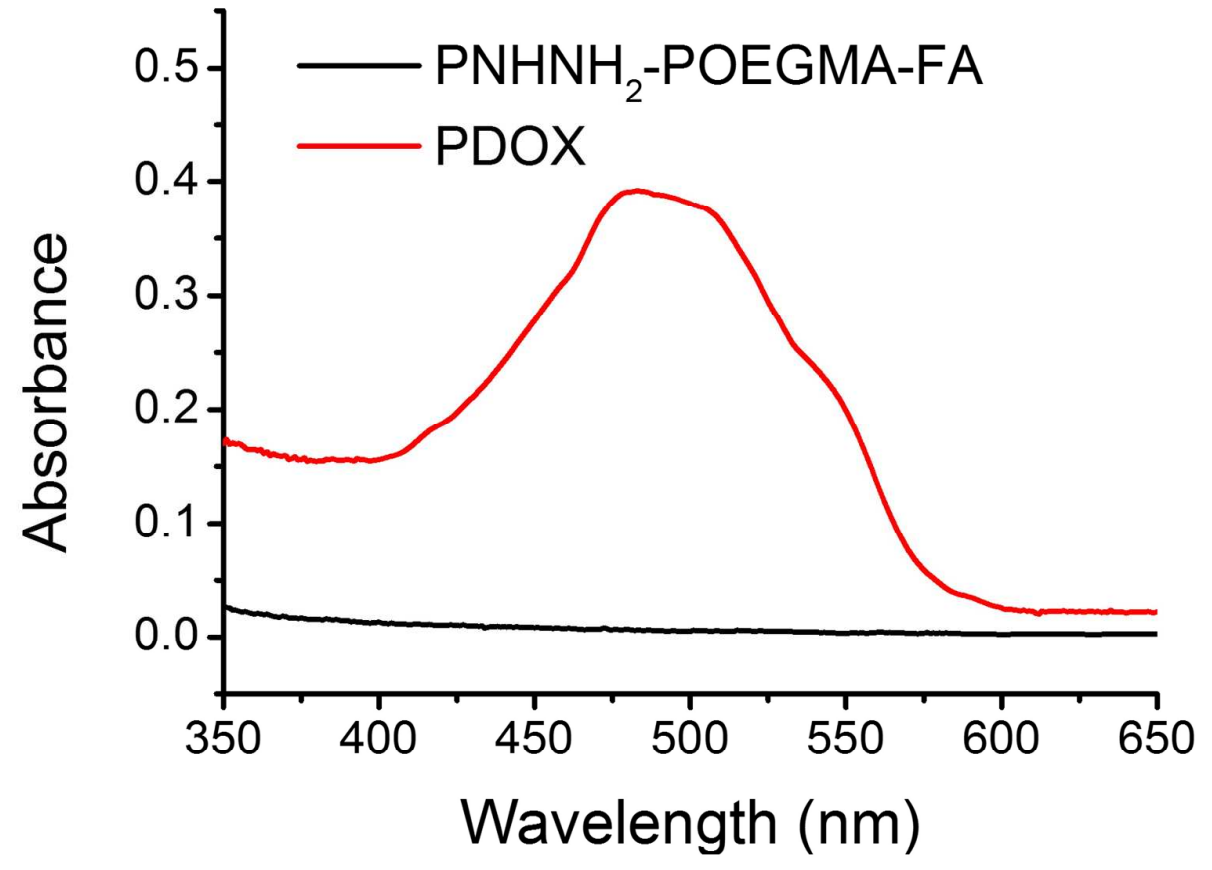

Figure S4. UV-Vis absorption spectra of $\mathrm{PNHNH}_{2}-b$-POEGMA-FA and PDOX in methanol. 


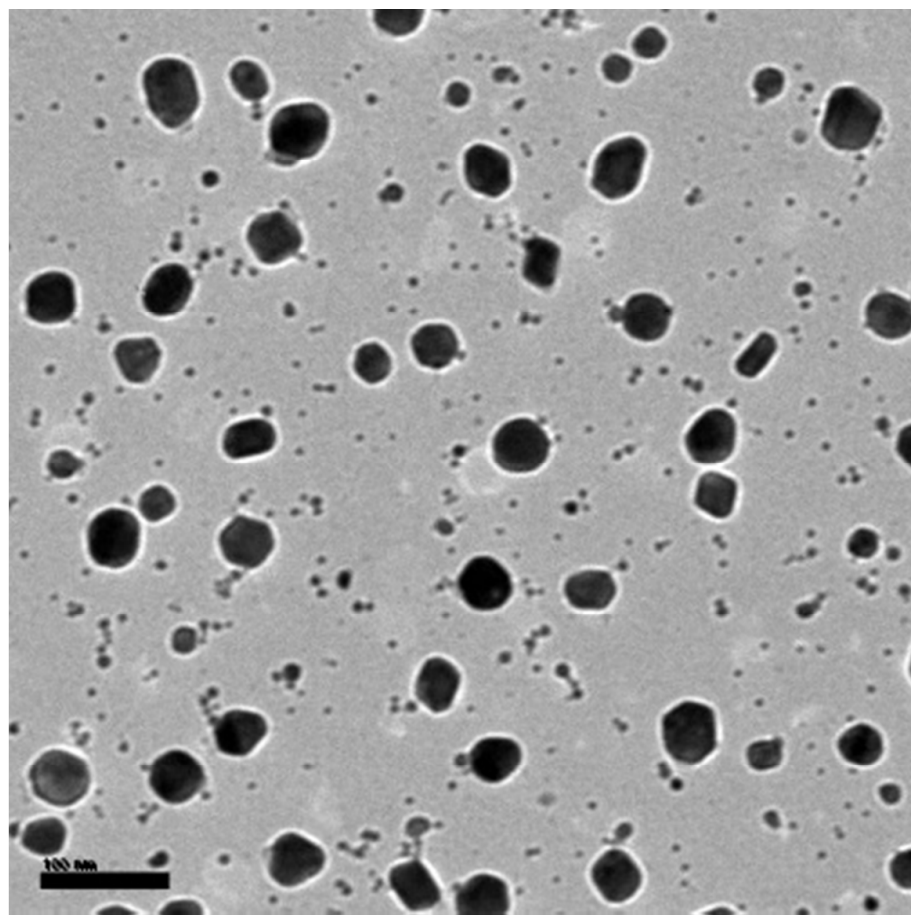

Figure S5. TEM image of PDOX prodrug nanoparticles. Scale bar $=100 \mathrm{~nm}$.
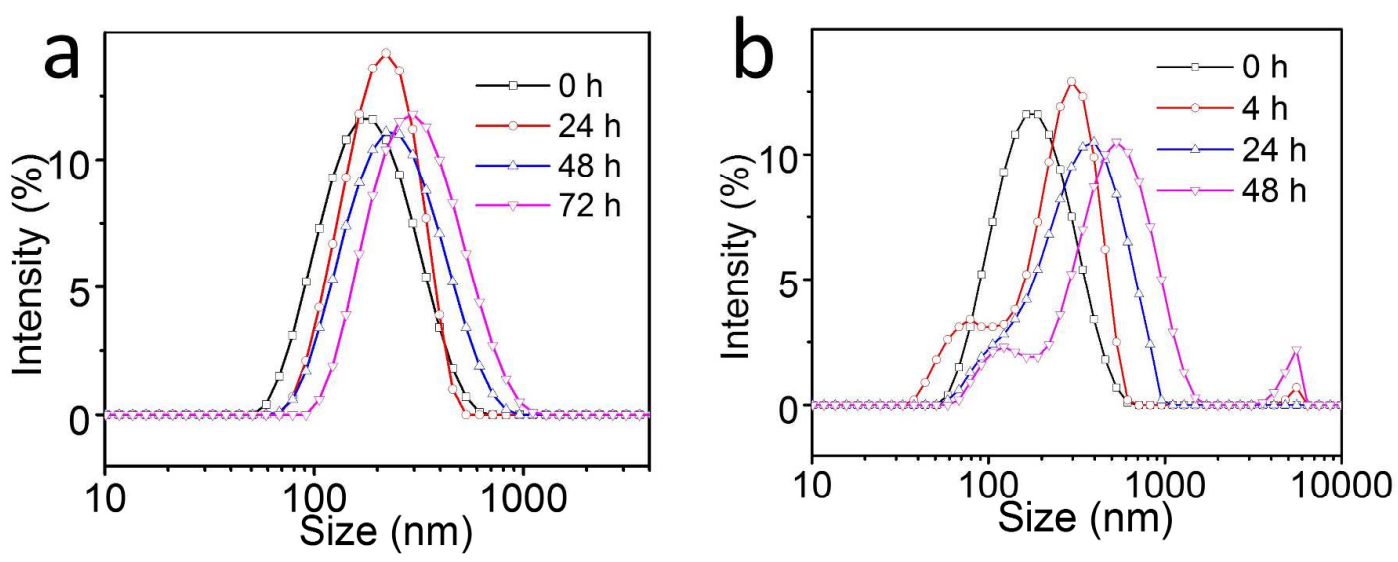

Figure S6. Changes of the size distribution of the PDOX/IR825 nanoparticles upon time in $\mathrm{pH}$ (a) 7.4 and (b) 5.0 buffer at $25^{\circ} \mathrm{C}$ monitored by DLS. 


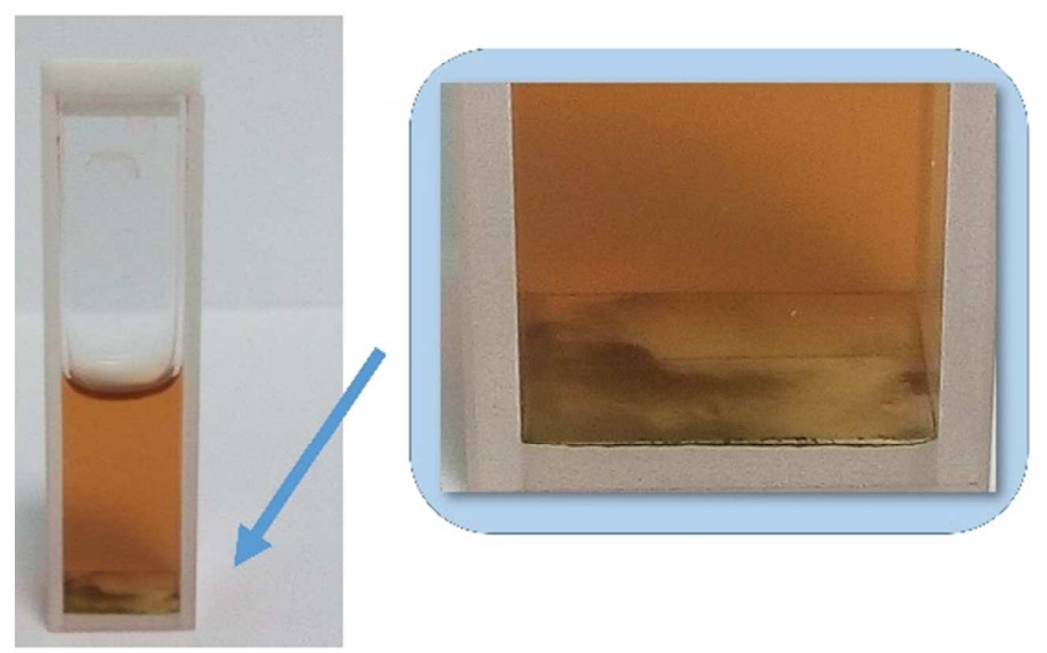

Figure S7. Photos of PDOX/IR825 suspension in phosphate buffer at $\mathrm{pH} 7.4$ (left) and PDOX/IR825 suspension treated with $0.1 \mathrm{M} \mathrm{HCl}$ overnight (right).
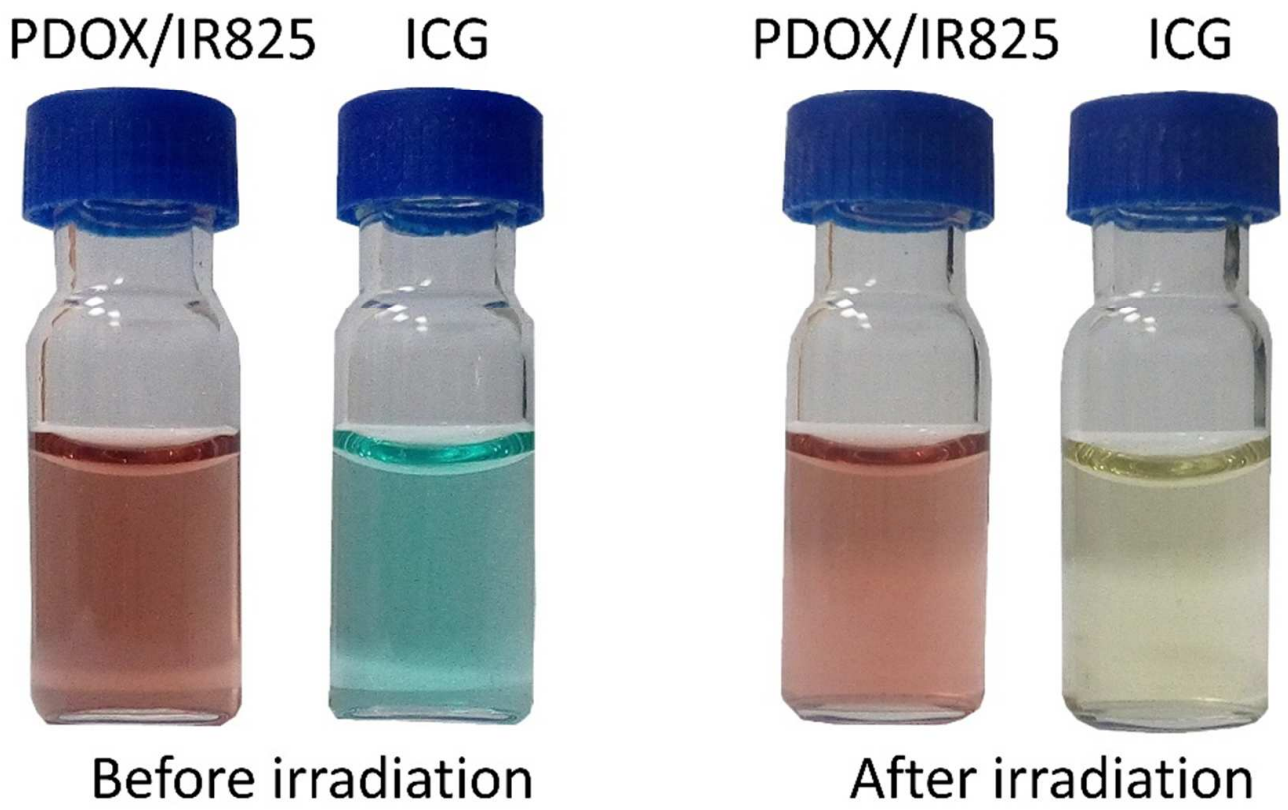

Figure S8. Photos of ICG solutions and PDOX/IR825 nanoparticle suspensions before and after 5 min of laser irradiation $\left(0.8 \mathrm{~W} \mathrm{~cm}^{-2}\right)$. 


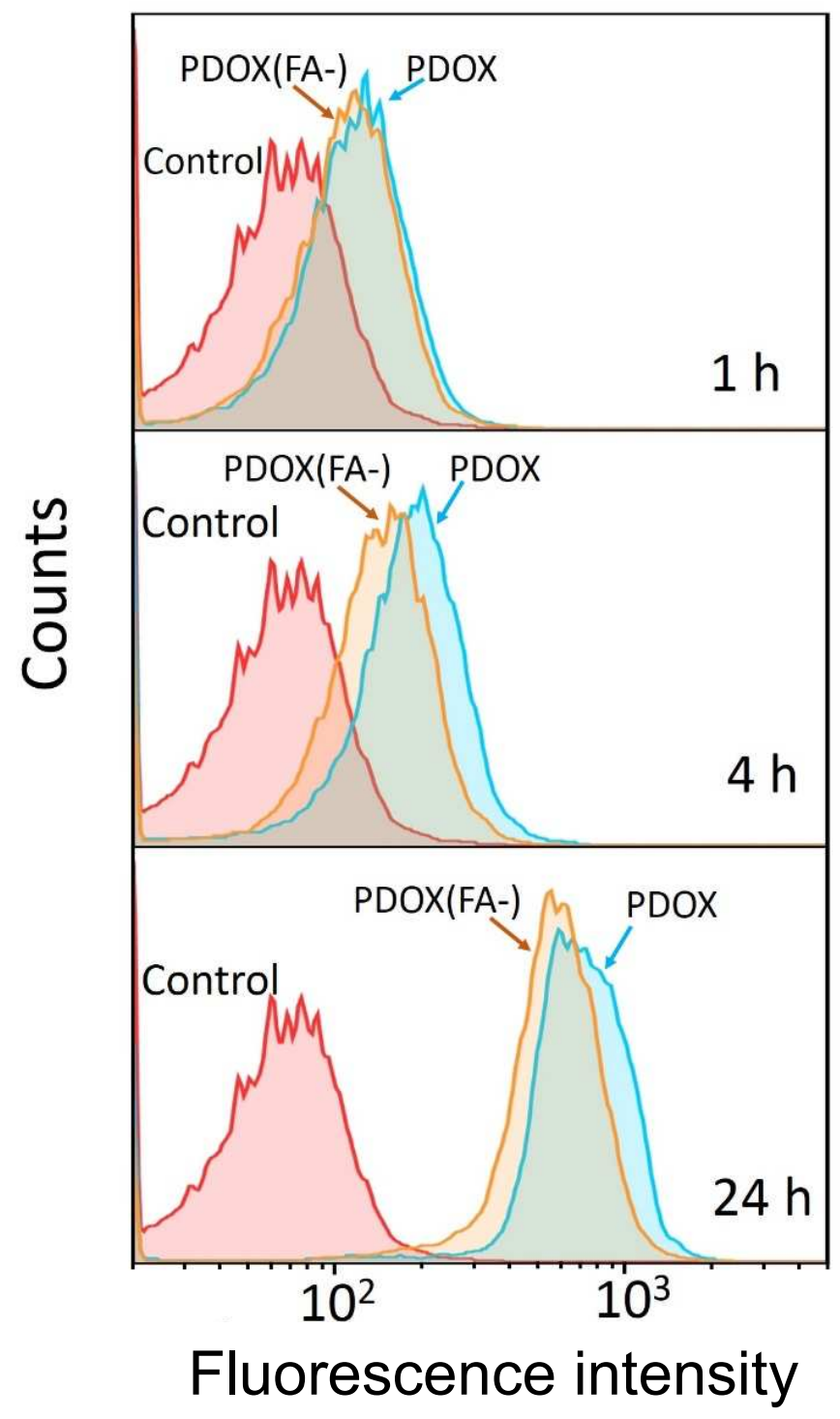

Figure S9. Flow cytometric analysis of the DOX fluorescence intensity in HeLa cells incubated with PDOX nanoparticles and polymeric DOX prodrug without the FA ligand PDOX(FA-) at the equivalent DOX concentration of $1 \mu \mathrm{g} \mathrm{mL}^{-1}$ for 1,4 and $24 \mathrm{~h}$. Control group refers to the cells without any treatment.

Table S2. IC50 values for free DOX and PDOX/IR825 without or with $808 \mathrm{~nm}$ laser irradiation against DOX-sensitive HeLa and DOX-resistant A2780/DOX ${ }^{\mathrm{R}}$ cells.

\begin{tabular}{ccc}
\hline \multirow{2}{*}{ Samples } & \multicolumn{2}{c}{$\mathrm{IC}_{50}\left(\mu \mathrm{g} \mathrm{mL}^{-1}\right)$} \\
\cline { 2 - 3 } & $\mathrm{HeLa}$ & $\mathrm{A} 2780 / \mathrm{DOX}^{\mathrm{R}}$ \\
\hline Free DOX & 3.9 & -- \\
PDOX/IR825 without irradiation & 6.7 & 12.6 \\
PDOX/IR825 with irradiation & 2.0 & 1.7 \\
\hline
\end{tabular}


Group 1: $\mathrm{H}_{2} \mathrm{O}, \mathrm{S} 1$

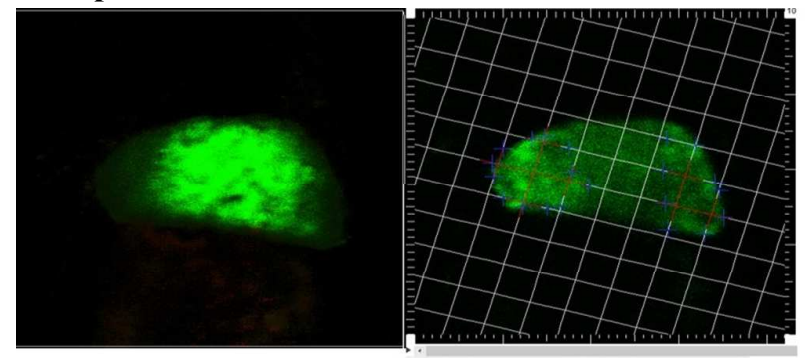

Group 1: $\mathrm{H}_{2} \mathrm{O}, \mathrm{S} 2$

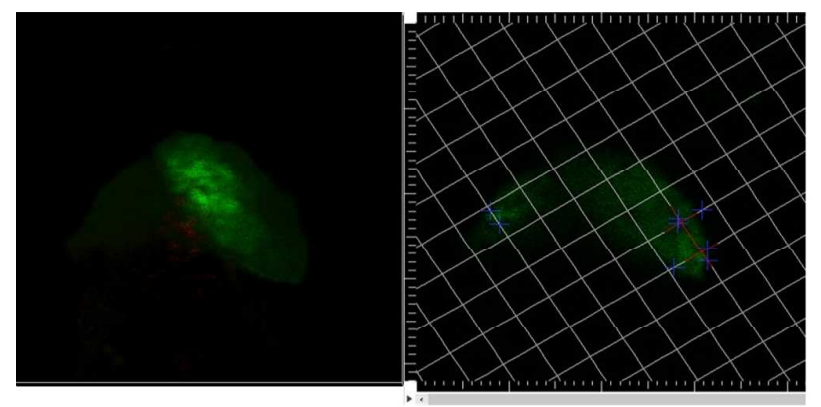

Group 1: $\mathrm{H}_{2} \mathrm{O}, \mathrm{S} 3$
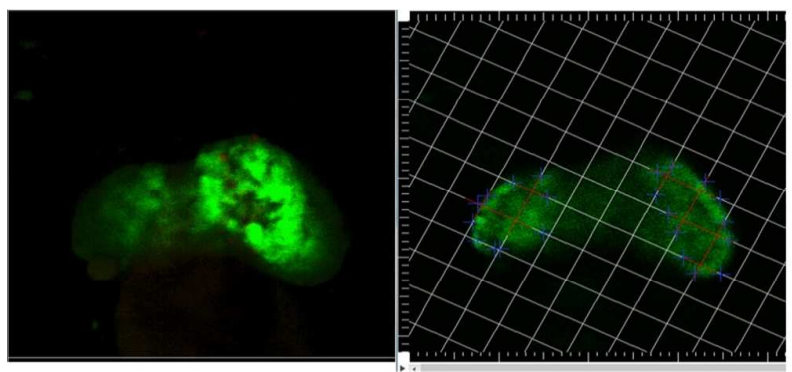

Group 1: $\mathrm{H}_{2} \mathrm{O}, \mathrm{S} 4$

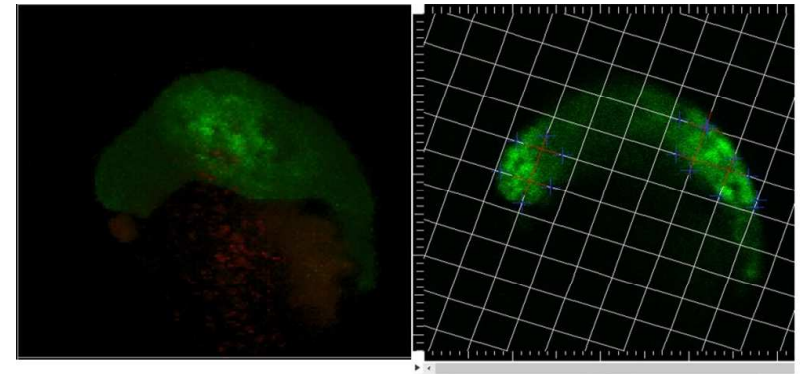

Group 1: $\mathrm{H}_{2} \mathrm{O}$, S5

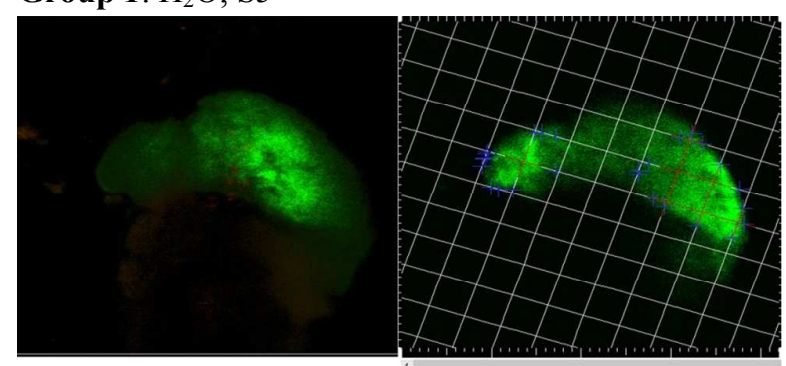

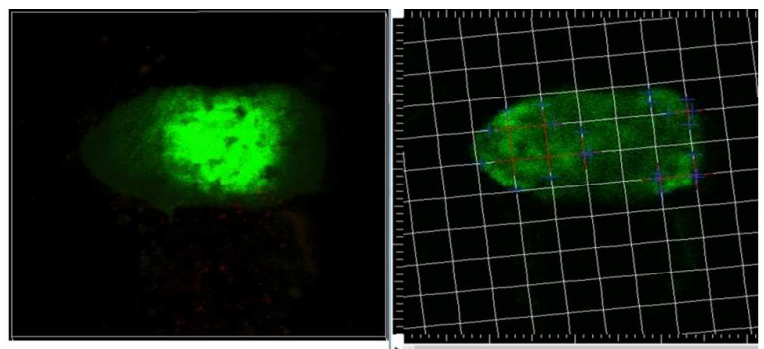
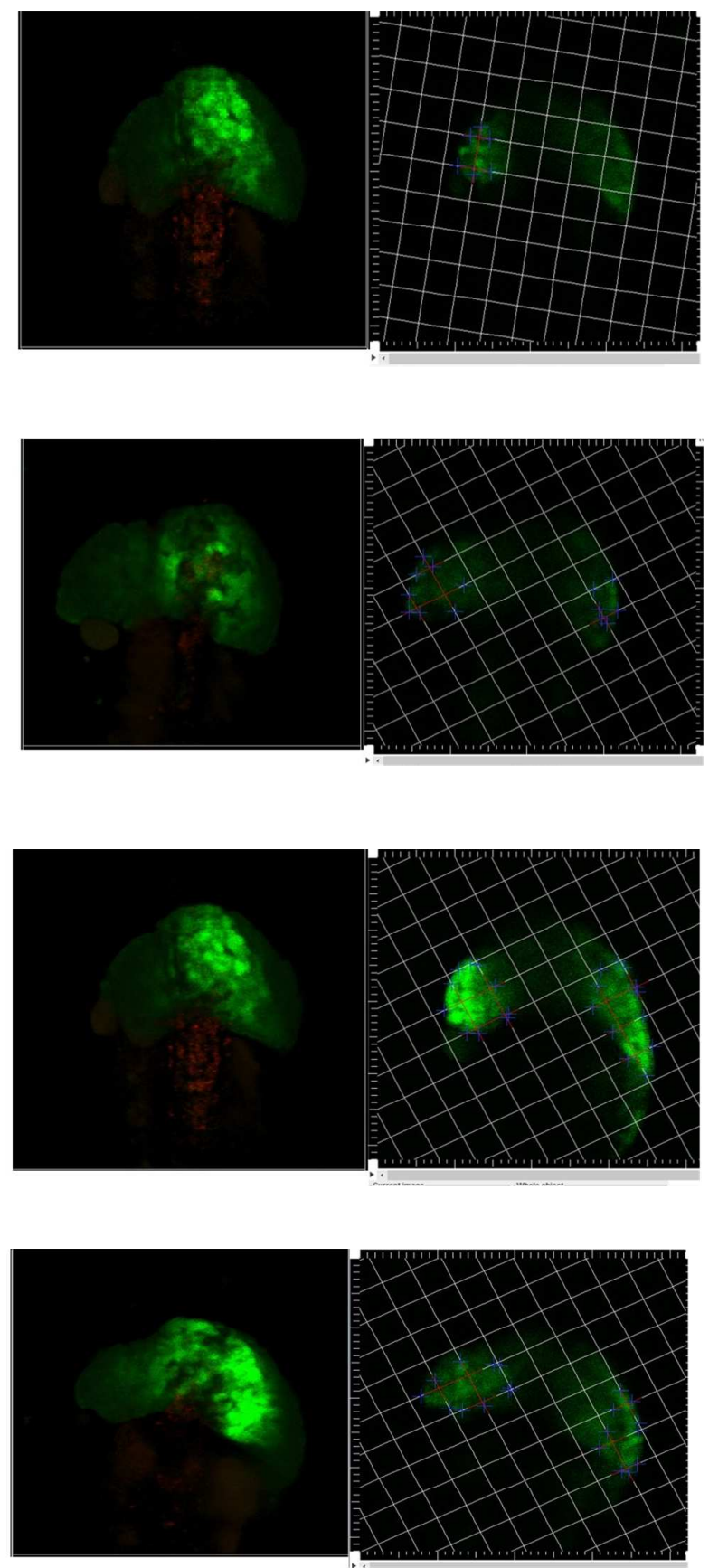
Group 2: free DOX, S1

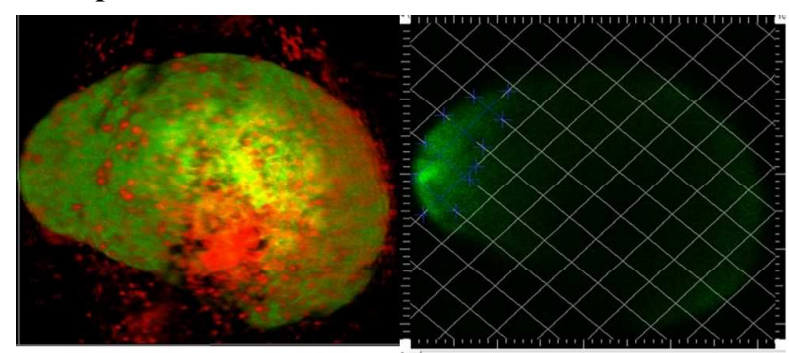

Group 2: free DOX, S2

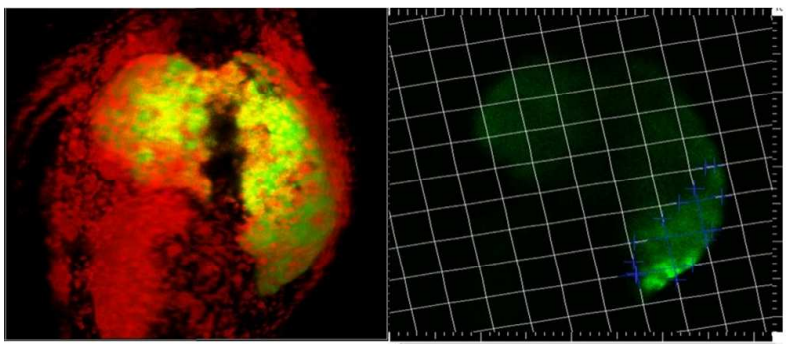

Group 2: free DOX, S3

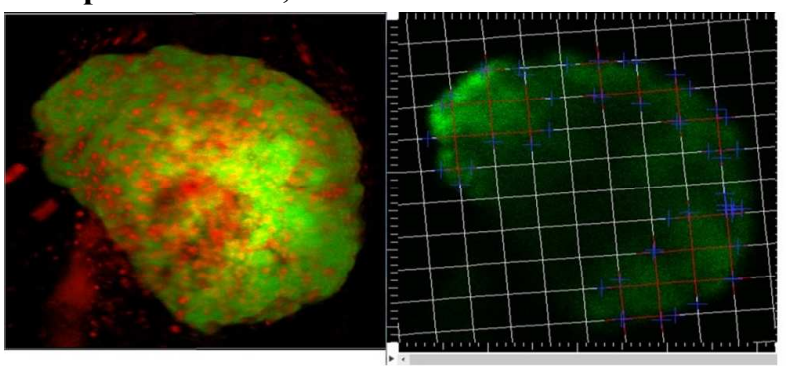

Group 2: free DOX, S4

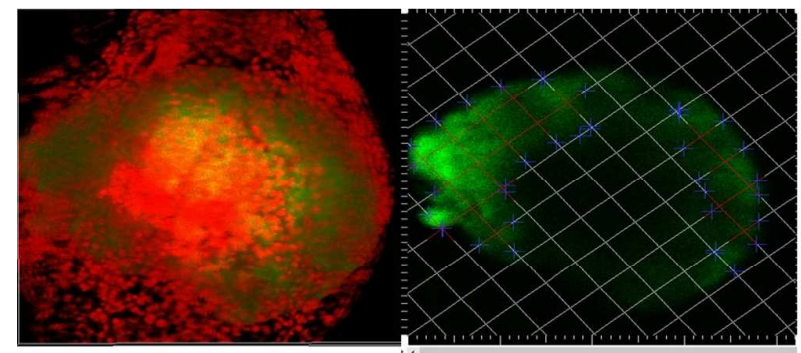

Group 2: free DOX, S5

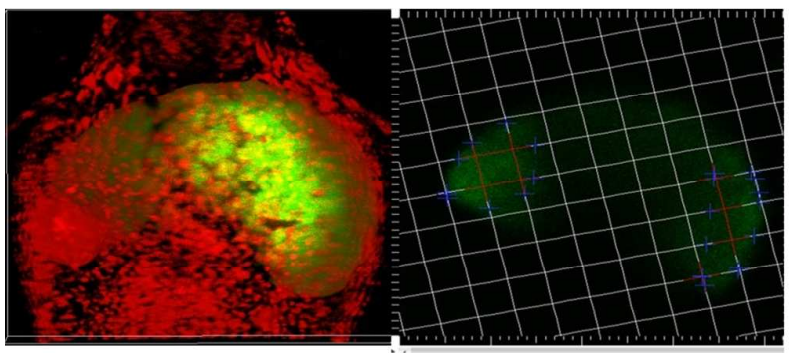

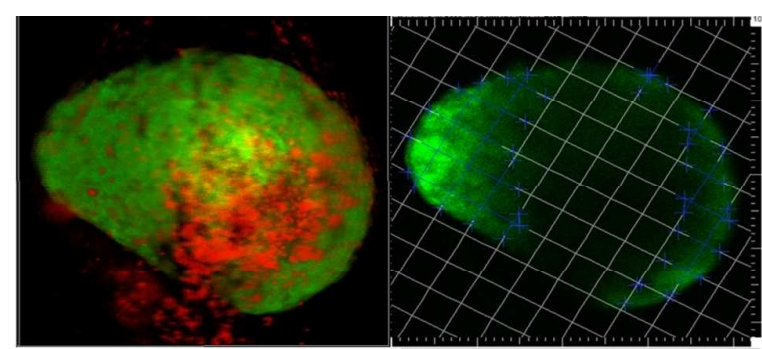
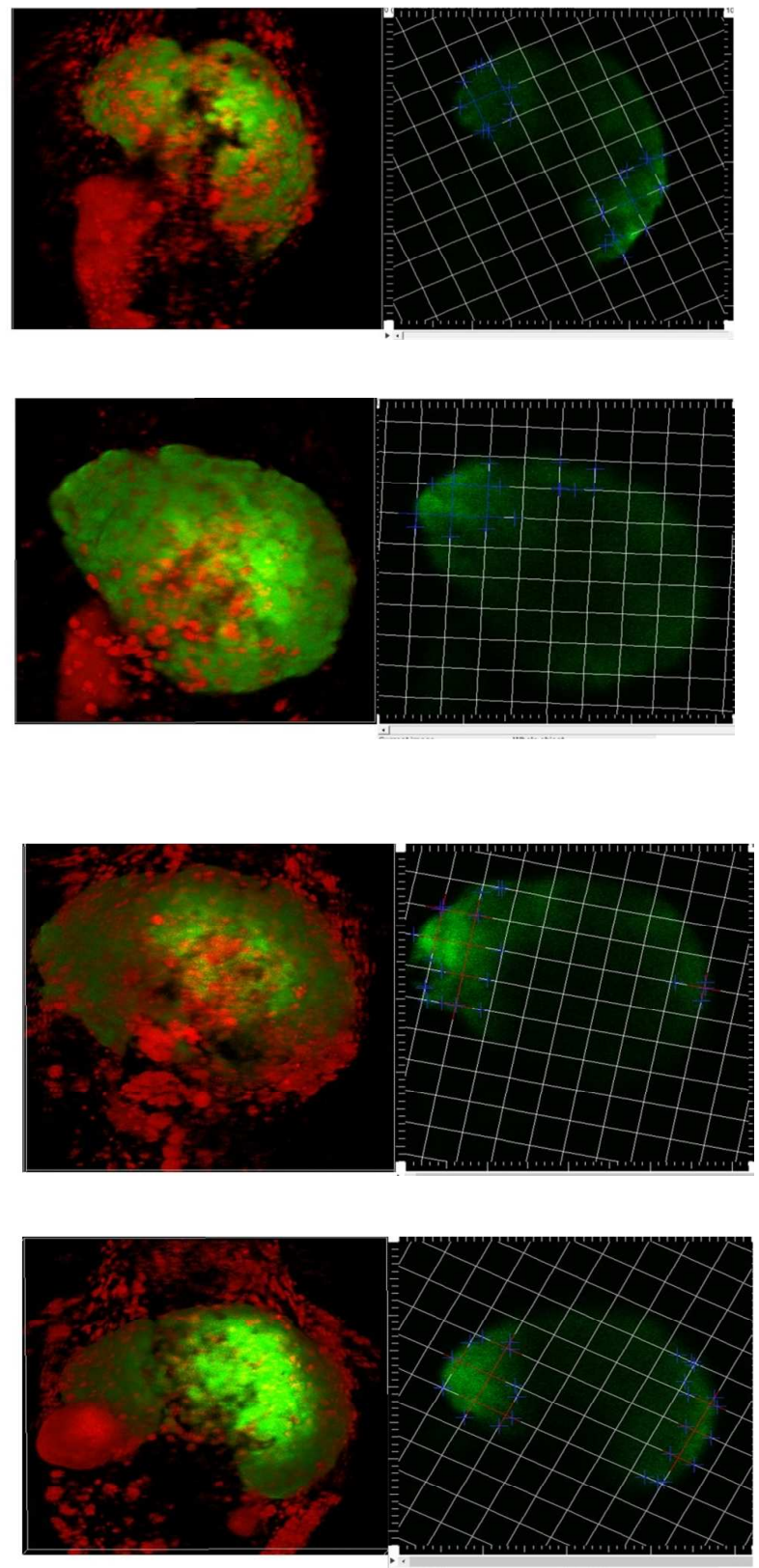
Group 3: PDOX/IR825 without NIR irradiation, S1

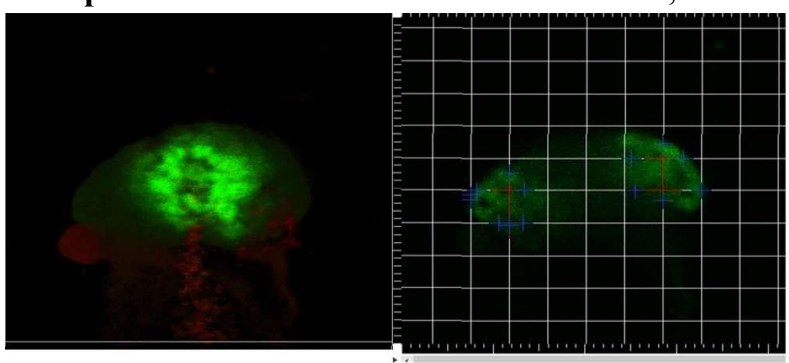

Group 3: PDOX/IR825 without NIR irradiation, S2

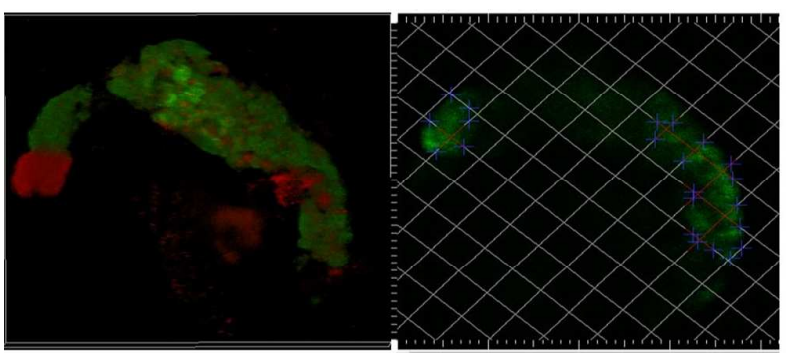

Group 3: PDOX/IR825 without NIR irradiation, S3

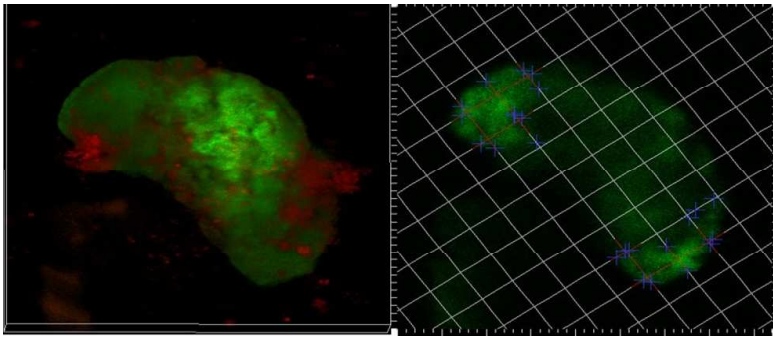

Group 3: PDOX/IR825 without NIR irradiation, S4

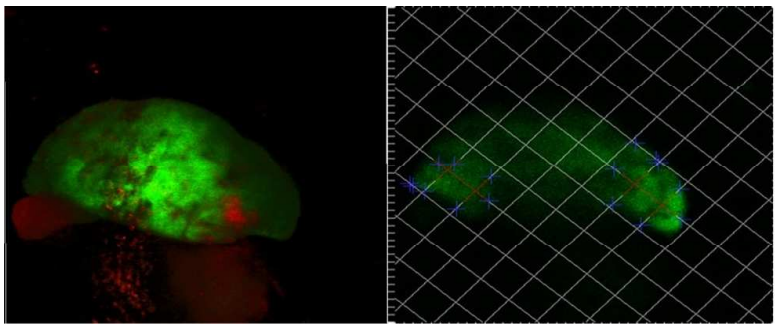

Group 3: PDOX/IR825 without NIR irradiation, S5

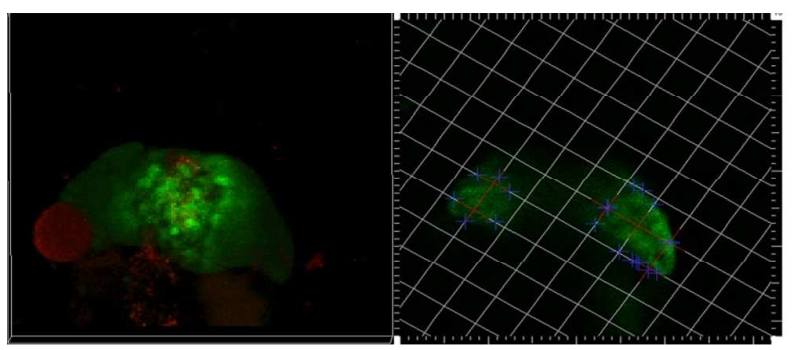

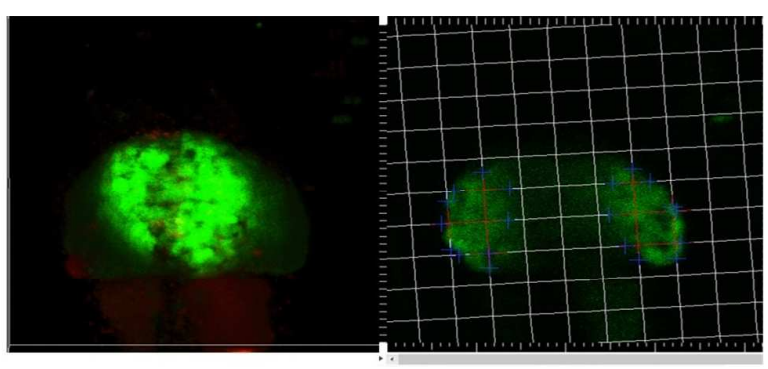
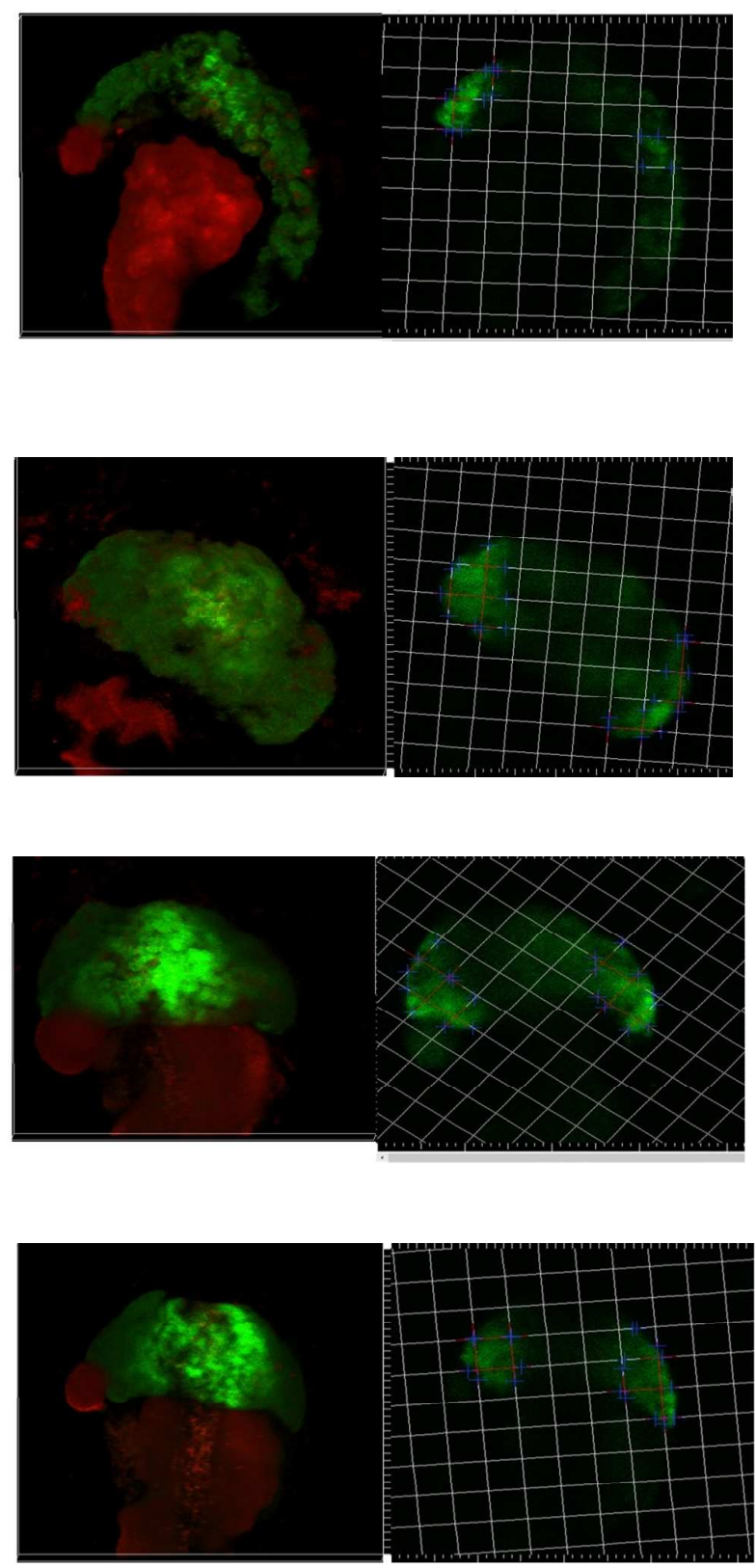
Group 4: PDOX/IR825 with NIR irradiation, S1
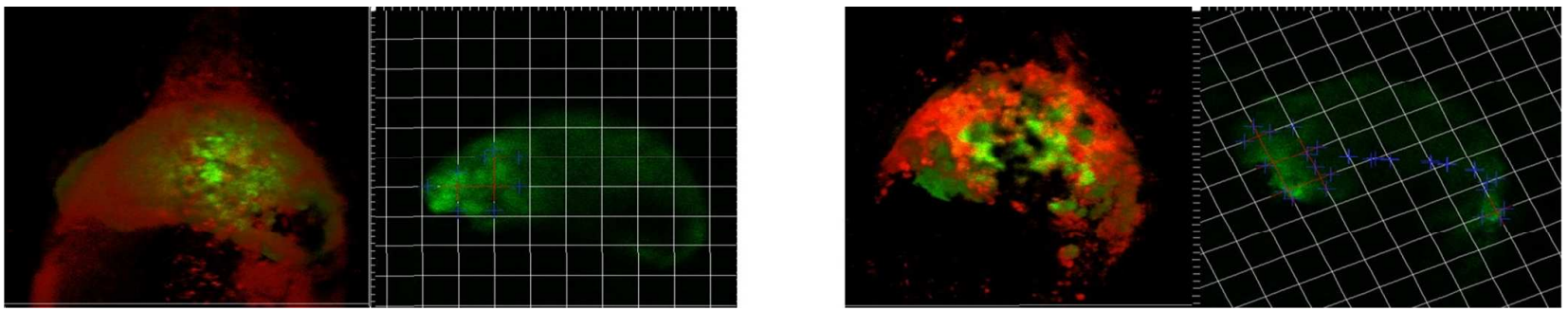

Group 4: PDOX/IR825 with NIR irradiation, S2
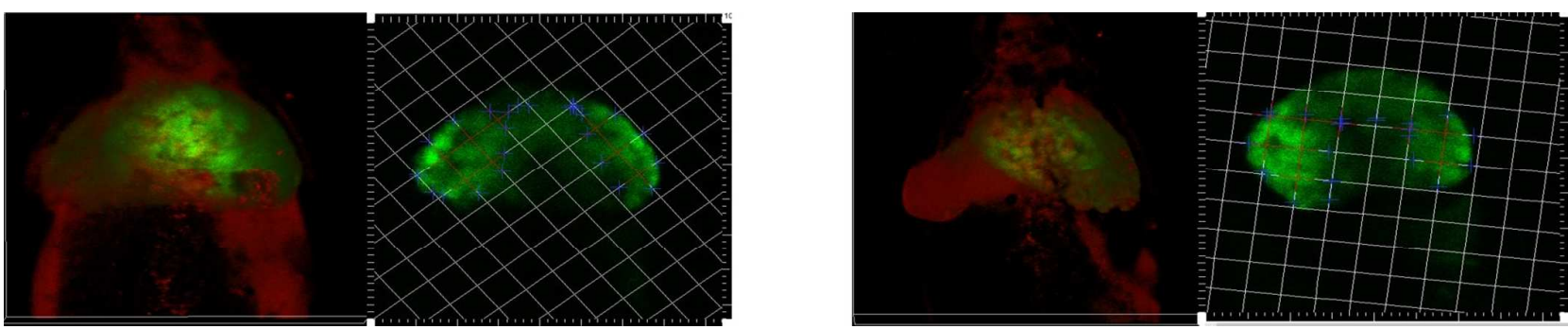

Group 4: PDOX/IR825 with NIR irradiation, S3
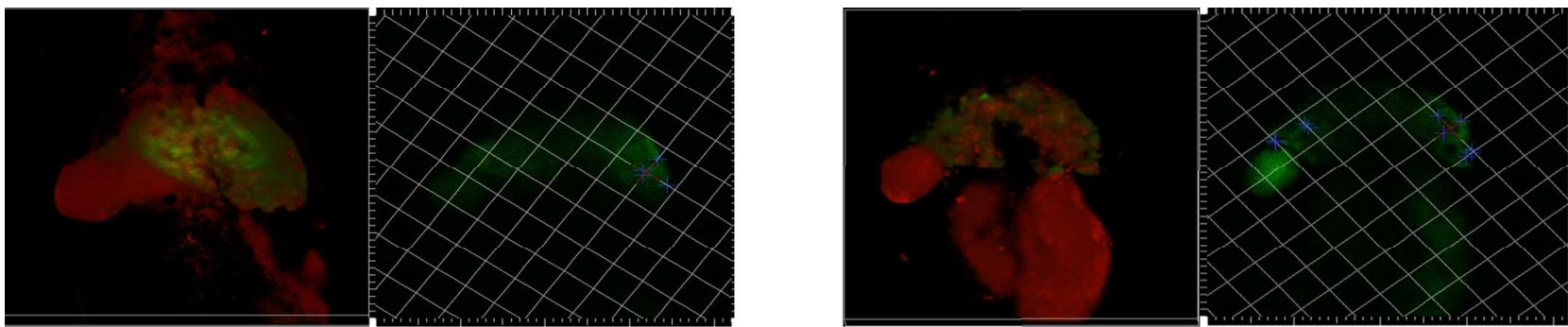

Group 4: PDOX/IR825 with NIR irradiation, S4
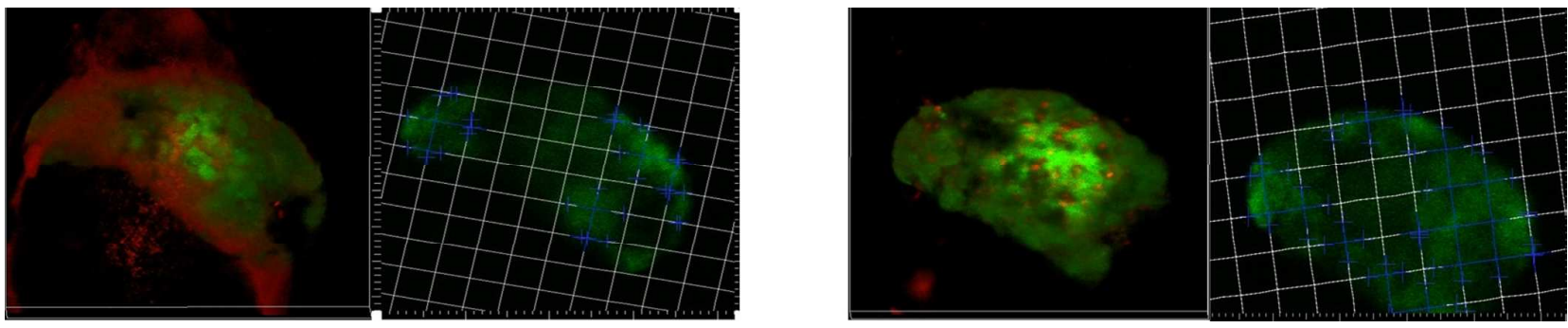

Group 4: PDOX/IR825 with NIR irradiation, S5
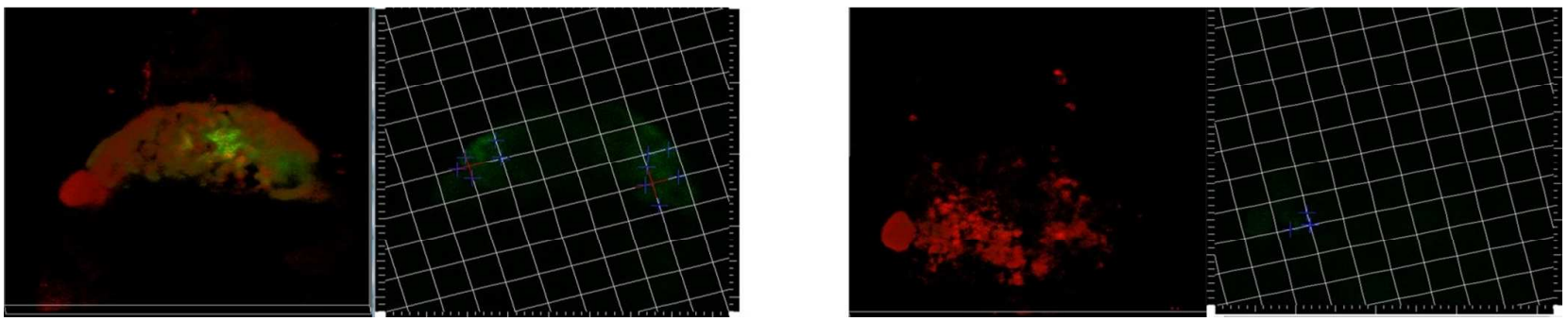

Figure S10. Tumor volume measurements from typical embryos in each sample group using Image J. Group 1: blank control treatment with $\mathrm{H}_{2} \mathrm{O}$, Group 2: treated with free DOX, Group 3: treated with PDOX/IR825 without NIR irradiation, Group 4: treated with PDOX/IR825 under NIR irradiation (5 min of $808 \mathrm{~nm}$ laser irradiation with the power density of $1.2 \mathrm{~W} \mathrm{~cm}^{-2}$ ). 